\title{
Gadolinium Enhancement of Atherosclerotic Plaque in the Middle Cerebral Artery: Relation to Symptoms and Degree of Stenosis
}

\author{
C.-W. Ryu, G.-H. Jahng, and H.S. Shin
}

\begin{abstract}
BACKGROUND AND PURPOSE: High-resolution MR imaging can depict intracranial arterial atherosclerotic plaques. Our aim was to evaluate the relationship between the degree of enhancement of MCA plaques on contrast-enhanced high-resolution MR imaging and ischemic stroke and stenosis severity.
\end{abstract}

MATERIALS AND METHODS: This study enrolled 36 patients diagnosed with moderate-to-severe atherosclerotic MCA stenosis. A contrast-enhanced T1-weighted volume isotropic turbo spin-echo acquisition sequence was acquired for assessing plaque enhancement. Plaque-to-CSF contrast ratio was calculated after the signal intensity of plaques at the stenotic segment was measured. Univariate comparison and multivariate logistic regression analyses were performed for symptomatic and asymptomatic groups to assess the relationship between symptomatic stenosis and independent variables, including plaque-to-CSF contrast ratio, degree of stenosis, and clinical risk factors. Plaque-to-CSF contrast ratio was compared between the moderate and severe stenosis groups.

RESULTS: Twenty-one patients had symptomatic MCA stenosis, and 15 had asymptomatic stenosis. The plaque-to-CSF contrast ratio was significantly higher in the symptomatic group than in the asymptomatic group (63.6 $\pm 10.6 \%$ versus $54.1 \pm 13.5 \%$, respectively; $P<.05)$. The degree of stenosis also differed significantly between the 2 groups $(P<.05)$. Multivariate analysis revealed that the degree of stenosis was the only independent predictor of ischemic stroke symptoms. The plaque-to-CSF contrast ratio of severe stenosis was significantly higher than that of moderate stenosis $(66.8 \pm 8.7 \%$ versus $55.9 \pm 12.8 \%$, respectively; $P<.05)$.

CONCLUSIONS: Plaque enhancement was significantly higher in patients with symptomatic plaques and may have been affected by the degree of stenosis. A difference in plaque enhancement according to the degree of stenosis has implications for understanding the development of intracranial atherosclerotic plaques.

ABBREVIATIONS: $C R$ = plaque-to-CSF contrast ratio; $\mathrm{Gd}$ = gadolinium; HR-MRI = high-resolution MR imaging; ICAD = intracranial atherosclerotic disease; $\mathrm{SI}=$ signal intensity; WASID = Warfarin Aspirin Symptomatic Intracranial Disease

l tracranial atherosclerotic disease (ICAD) is now considered the most common cause of ischemic stroke worldwide. ${ }^{1,2}$ It is a challenge for many clinicians to assess vulnerable regions of intracranial artery stenosis by using in vivo methods. As a result, there has been a recent increase in interest in techniques to depict the state of the intracranial artery wall by using high-resolution MR imaging (HR-MRI) with a high magnetic field.

Received January 21, 2014; accepted after revision May 30.

From the Departments of Radiology (C.-W.R., G.-H.J.) and Neurosurgery (H.S.S.), Kyung Hee University Hospital at Gangdong, Kyung Hee University School of Medicine, Seoul, Korea.

This work was supported by a grant from Kyung Hee University in 2012 (KHU-20120760)

Please address correspondence to Chang-Woo Ryu, MD, Department of Radiology, Kyung Hee University Hospital at Gangdong, 149 Sangil-dong, Gangdong-gu, Seoul 134-727, Korea; e-mail: md.cwryu@gmail.com

http://dx.doi.org/10.3174/ajnr.A4038
Inflammation increases vulnerability to plaque rupture by facilitating neovascularization within the plaque and increasing endothelial permeability. Strong contrast enhancement of arterial plaques on MR imaging suggests the presence of a vascular supply and increased endothelial permeability, which facilitate the entry of contrast agents from the blood plasma. ${ }^{3-6}$ Several studies have shown that gadolinium (Gd) enhancement of extracranial carotid plaques on MR imaging is associated with plaque neovascularization or increased levels of serum inflammatory markers such as C-reactive protein. ${ }^{7-10}$ An association between carotid plaque enhancement and clinical symptoms has been observed in several recent investigations by using Gd-enhanced MR imaging., ${ }^{9,11}$

In contrast to the numerous investigations that have been performed on the carotid artery, only a few studies performed on a small number of patients have focused on the clinical significance of Gd enhancement of plaques in the setting of ICAD. ${ }^{12-14}$ These studies identified a significant association between plaque en- 
hancement on ICAD and ischemic events. However, there is some debate about whether contrast enhancement is a good predictor of intracranial stenosis in ischemic stroke. Klein et $\mathrm{al}^{15}$ expressed doubt about the relationship between plaque enhancement and stroke because they found stationary enhancement of basilar plaques regardless of the time elapsed after an acute event.

Several factors are known to be related to ischemic stroke in the territory of the stenotic segment. ${ }^{16,17}$ In particular, the severity of stenosis is a strong predictor of subsequent stroke in the territory of the symptomatic stenotic artery. To assess whether Gd enhancement of plaques at the stenotic segment is valuable as a predictor of ischemic stroke, an unbiased assessment of the influence of other independent factors, including the degree of stenosis, should be undertaken. Thus, the purpose of this study was to evaluate whether Gd enhancement of plaques in ICAD is associated with clinical symptoms, independent of other predictive factors for ischemic stroke risk.

\section{MATERIALS AND METHODS Subjects}

This study was approved by the local ethics committee. Patients with acute neurologic symptoms underwent an acute stroke MR imaging protocol and postcontrast HR-MRI between August 2011 and October 2012. Before MR imaging, all subjects provided informed consent to undergo MR imaging with the use of contrast media. The radiologic findings from the MR imaging were reviewed, and those subjects who had unilateral M1 stenosis of $>70 \%$ on TOF-MRA and $\geq 2$ risk factors for atherosclerosis were enrolled in the study.

After reviewing patient medical records and MR imaging findings, we excluded patients who met the following criteria: 1) a high risk of carotid artery-to-artery embolism (ipsilateral ICA stenosis of $>50 \%$ ); 2) a high risk of cardioembolism (eg, atrial fibrillation, prosthetic cardiac valve); 3 ) vascular disease of other clinically and/or radiologically suspected etiology, such as Moyamoya disease, vasculitis, or dissection; 4) known coagulopathies (eg, protein C or S deficiency, antiphospholipid antibody syndrome); 5) ipsilateral acute lacunar infarction (single acute ischemic lesion of $<2 \mathrm{~cm}$ at the basal ganglia or the corona radiata) at sites of MCA stenosis; 6) T1 hyperintensity within the stenotic MCA representing intraplaque hemorrhage and/or intraluminal thrombus on MPRAGE MR imaging; and 7) poor MRA or HR-MRI image quality, making the image nondiagnostic or difficult to analyze quantitatively.

Forty-two subjects met all inclusion criteria. However, 4 patients with ipsilateral cavernous ICA stenosis and 2 patients with ipsilateral cervical carotid stenosis were excluded. None of the patients were excluded due to either intraluminal thrombus or poor image quality. In total, 36 subjects (mean age, $68.69 \pm 12.21$ years; 17 males) were enrolled in this study.

\section{MR Imaging}

MR imaging was performed on a $3 \mathrm{~T}$ MR imaging system (Achieva; Philips Healthcare, Best, the Netherlands) with a 16channel phased array neurovascular coil. The MR imaging stroke protocol included the following: T1-weighted MPRAGE sagittal imaging with presaturation of inflowing blood, axial DWI, T2- weighted FLAIR axial imaging, TOF-MRA, and contrast-enhanced neck MRA. To evaluate plaques in the MCA, we added to the stroke protocol postcontrast HR-MRIs that were obtained axially at the circle of Willis, as shown on TOF-MRA. Contrastenhanced HR-MRIs were acquired with 3D T1-weighted volume isotropic turbo spin-echo acquisition axial imaging with presaturation of inflowing blood to obtain black-blood images. Scans were started with an approximately 4-minute delay time, including the scan time for contrast-enhanced neck MRA after injection of contrast media. We used the following HR-MRI parameters: $\mathrm{TR}=350 \mathrm{~ms}, \mathrm{TE}=19.51 \mathrm{~ms}$, flip angle $=90^{\circ}$, refocusing angle $=$ $120^{\circ}$, acquisition matrix $=240 \times 240, \mathrm{FOV}=120 \times 120,3 \mathrm{D}$ slab thickness $=13 \mathrm{~mm}$, in-plane pixel size $=0.5 \times 0.5 \mathrm{~mm}$, overcontiguous section thickness $=2 \mathrm{~mm}$, reconstruction matrix $=$ $352 \times 352$, reconstruction voxel size $=0.34 \times 0.34 \times 1 \mathrm{~mm}$, reconstruction section thickness $=1 \mathrm{~mm}$, foldover direction $=$ anteroposterior, signal average $=2$, TSE factor $=3$, sensitivity encoding factor $=2$ for the phase-encoding direction and 1 for the section-selection direction, number of stacks $=2$ for each of the 13 sections, transverse presaturation thickness $=80 \mathrm{~mm}$, total scan time $=184$ seconds.

\section{Clinical Data Assessment}

Subjects were classified as having either symptomatic stenosis or asymptomatic stenosis according to the presence of recent ischemic stroke consistent with MCA stenosis. Symptomatic stenosis was defined as a diffusion-restrictive lesion seen on DWI in the territory of the stenotic MCA with a corresponding acute neurologic deficit within 2 weeks before MR imaging. Asymptomatic stenosis was diagnosed in patients with nonspecific neurologic symptoms (headache, dizziness, and so forth) that were not localized to the ipsilateral side of the MCA stenosis and who had no observations of any ischemic lesion on DWI. Patients with acute ischemic lesions localized to the territory of the posterior circulation, the anterior cerebral artery, or the contralateral MCA were considered to have asymptomatic MCA stenosis.

Clinical data, including basic demographics and risk factors for atherosclerosis, namely diabetes, hypertension, dyslipidemia, current smoking, obesity, and history of coronary disease, were also recorded.

\section{MR Imaging Assessment}

According to the distribution of acute ischemic lesions on DWI, infarctions in the symptomatic group were classified as borderzone, striatocapsular, or pial embolic, according to the previously published topographic definition of DWI. ${ }^{18}$ The degree of MCA stenosis was classified as severe or moderate according to TOFMRA findings. Severe stenosis was defined as a flow signal defect at the stenotic segment (Fig 1). Moderate stenosis was defined as stenosis of $>70 \%$ that did not meet the criteria for severe stenosis (Fig 2). Although MRA is considered a relatively accurate diagnostic tool for grading intracranial artery stenosis, ${ }^{19,20}$ interpretations of MRA generally show a tendency to overestimate the degree of stenosis. The criterion for moderate stenosis based on MRA was therefore set to $>70 \%$, thus modifying the Warfarin Aspirin Symptomatic Intracranial Disease (WASID) angiographic criterion for moderate stenosis $(>50 \%) .{ }^{21}$ The degree 

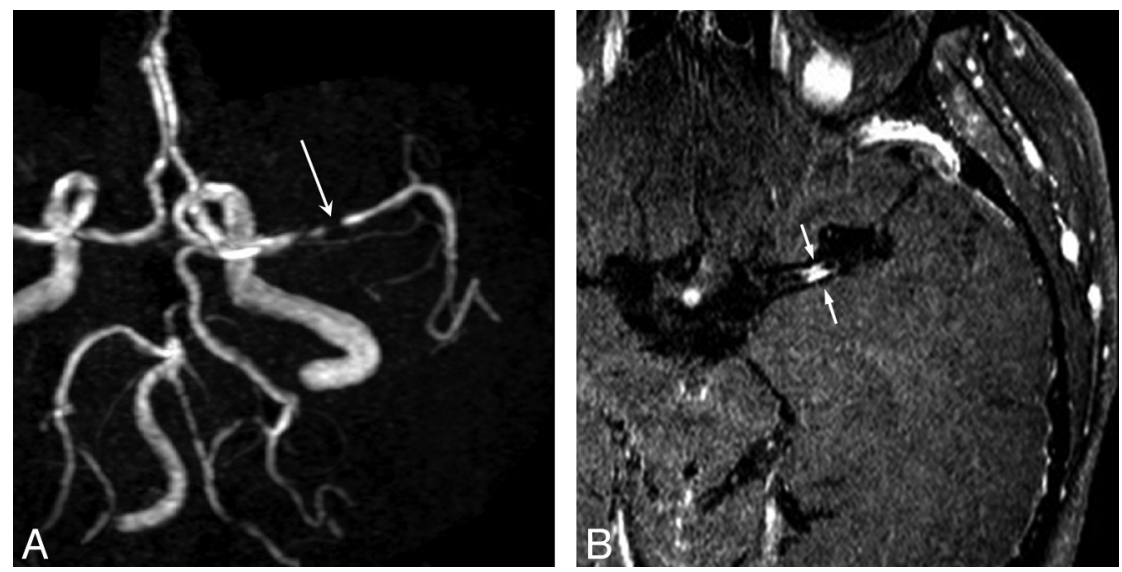

variate methods (2-sample $t$ test or Fisher exact test). Stepwise multivariate logistic regression analysis was used to assess how independent variables, including clinical risk factors, degree of stenosis, and CR, were related to the presence of acute symptoms. CR was compared between moderate and severe stenosis cases by using the 2 -sample $t$ test. A $P$ value $<.05$ was considered statistically significant.

\section{RESULTS}

Of the 36 subjects enrolled in our study, 21 (mean age, $69.7 \pm 11.9$ years; 10 men) were classified as having symptomatic ste-

FIG 1. A 73-year-old man with severe MCA stenosis (A, long arrow) who presented with multifragmented infarctions at the ipsilateral MCA territory. Postcontrast MR image shows attenuated enhancement of the plaque ( $B$, small arrows).
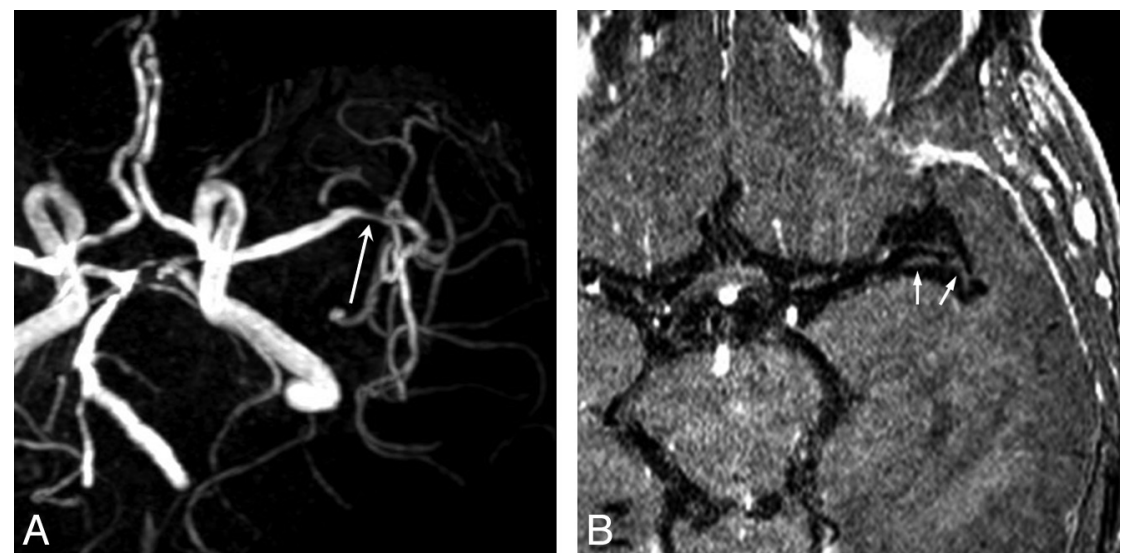

FIG 2. An 88-year-old woman with asymptomatic moderate MCA stenosis ( $A$, long arrow) has relatively little enhancement of MCA plaques (B, small arrows).

of stenosis was measured by the WASID method ${ }^{22}$ by careful review and consensus of 2 raters (a neuroradiologist and a neurosurgeon).

The degree of enhancement was quantified by drawing ROIs on the contrast-enhanced HR-MRI. ROIs were ovoid or linear and placed to cover the plaques in a manner that was consistent with the stenotic segment of the MCA on MRA by a neuroradiologist. To increase the reliability of the ROIs, the rater measured the signal intensity (SI) 3 times with a region-of-interest drawing and then averaged the SI values. The plaque-to-CSF contrast ratio (CR) was calculated by using the following equation ${ }^{23}$ :

$$
\mathrm{CR}_{\text {plaque-to-CSF }}(\%)=\left(\mathrm{SI}_{\text {plaque }}-\mathrm{SI}_{\mathrm{CSF}}\right) \times 100 /\left(\mathrm{SI}_{\text {plaque }}+\mathrm{SI}_{\mathrm{CSF}}\right) \text {, }
$$

where $\mathrm{SI}_{\text {plaque }}$ is plaque $\mathrm{SI}$ and $\mathrm{SI}_{\mathrm{CSF}}$ is CSF SI measured by manual drawing of the round region of interest at the suprasellar cistern or Sylvian cistern. When drawing the region of interest to measure $\mathrm{SI}_{\mathrm{CSF}}$, the neuroradiologist was careful not to include any vessels within the cisternal space.

\section{Statistical Analyses}

To evaluate the difference between symptomatic and asymptomatic groups, we compared radiologic findings (stenosis degree and CR) and clinical parameters between the 2 groups by using uni- nosis and 15 (mean age, $67.3 \pm 12.9$ years; 7 men) were classified as having asymptomatic stenosis. Among the 21 cases of symptomatic stenosis, 10 were subcategorized as pial embolic infarctions, 7 were considered borderzone infarctions, and 4 were striatocapsular infarctions. Eleven of the symptomatic cases (52\%) had moderate stenosis, and $10(48 \%)$ had severe stenosis. The mean CR for symptomatic stenosis was $63.64 \pm 10.56 \%$. Among 15 subjects with asymptomatic stenosis, 8 subjects had acute infarctions in inconsistent areas and 7 did not have any ischemic lesions or symptoms. Thirteen subjects $(87 \%)$ were considered to have moderate stenosis, and 2 (13\%) had severe stenosis. The mean CR for asymptomatic stenosis was $54.09 \pm 13.49 \%$.

Univariate comparisons of radiologic and clinical variables showed that the CR of the symptomatic stenosis group was significantly higher than that of the asymptomatic stenosis group ( $P=.029 ; 95 \% \mathrm{CI}, 0.99-17.27)$ (Fig 3A). The Fisher exact test showed that the frequency of severe stenosis was significantly higher in the symptomatic group than in the asymptomatic group $(P=.039)$. Demographic variables and atherosclerotic risk factors were not significantly different between the 2 groups (Table). Multivariate logistic regression analysis revealed that the severity of MCA stenosis was the only statistically significant independent predictor of ischemic stroke symptoms (odds ratio $=7.15 ; 95 \% \mathrm{CI}, 1.28-39.83 ; P=.025$ ).

In total, there were 24 patients with moderate stenosis and 12 with severe stenosis. The mean CRs of patients with moderate and severe stenosis were $55.91 \pm 12.82 \%$ and $66.78 \pm 8.70 \%$, respectively. The CR for patients with severe stenosis was significantly higher than that of those with moderate stenosis $(P=.010$; 95\% CI, 2.74-19.01) (Fig 3B).

\section{DISCUSSION}

In this study, Gd enhancement of MCA plaques was associated with the degree of stenosis and ischemic stroke in the territory of the stenotic segment; the degree of stenosis was also associated with clinical symptoms. Although there was a positive relation- 

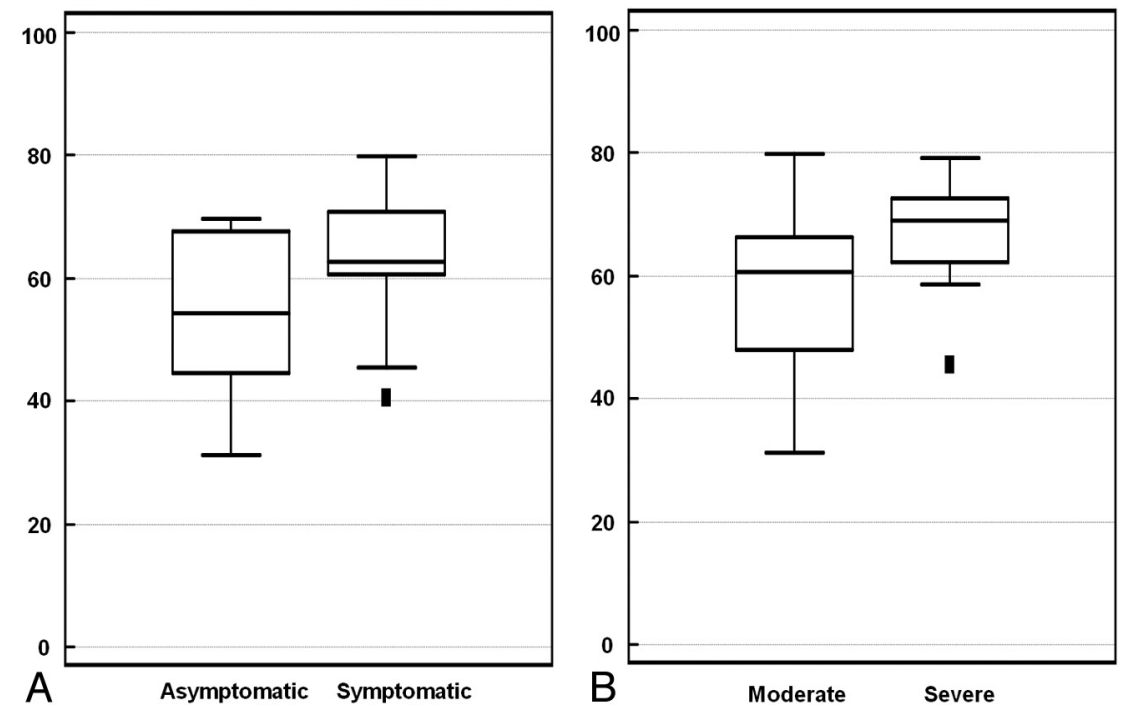

FIG 3. A, Comparison of the plaque-to-CSF contrast ratio between asymptomatic and symptomatic stenosis groups. Mean CRs for asymptomatic and symptomatic stenosis are $54.09 \pm$ $13.49 \%$ and $63.64 \pm 10.56 \%$, respectively. B. Comparison of CRs between moderate and severe MCA stenosis. Mean CRs for moderate and severe stenosis are $55.91 \pm 12.82 \%$ and $66.78 \pm$ $8.70 \%$, respectively.

Comparison of asymptomatic and symptomatic stenosis groups ${ }^{a}$

\begin{tabular}{lccr}
\hline \multicolumn{1}{c}{ Characteristics } & $\begin{array}{c}\text { Symptomatic } \\
\text { Stenosis }(\boldsymbol{n}=\mathbf{2 1})\end{array}$ & $\begin{array}{c}\text { Asymptomatic } \\
\text { Stenosis }(\boldsymbol{n}=15)\end{array}$ & $\boldsymbol{P ~ V a l u e ~}^{\mathbf{b}}$ \\
\hline Age (yr) & $69.7 \pm 11.9$ & $67.3 \pm 12.9$ & .58 \\
Sex, male & 10 & 7 & 1.00 \\
Risk factor & & & \\
$\quad$ HTN & $13(62 \%)$ & $12(80 \%)$ & .29 \\
DM & $8(38 \%)$ & $8(53 \%)$ & .49 \\
Dyslipidemia & $12(57 \%)$ & $11(73 \%)$ & .48 \\
Current smoking & $8(38 \%)$ & $4(27 \%)$ & .72 \\
Obesity & $11(52 \%)$ & $5(33 \%)$ & .32 \\
CHD & $2(10 \%)$ & $2(13 \%)$ & 1.00 \\
MCA stenosis & & & .09 \\
Site & 12 right, 9 left & 4 right, 11 left & $<.05$ \\
Degree & 11 moderate, 10 severe & 13 moderate, 2 severe & $<.05$ \\
CR $(\%)$ & $63.64 \pm 10.56$ & $54.09 \pm 13.49$ & \\
\hline
\end{tabular}

Note:-CHD indicates coronary heart disease; DM, diabetes mellitus; HTN, hypertension.

a Values are presented as numbers (\%). Age and CR are presented as means.

${ }^{b} P$ values were calculated by the $t$ test and Fisher exact test.

' The degree of stenosis was the only independent variable that survived multivariate logistic regression analysis (odds ratio $=7.15 ; 95 \% \mathrm{Cl}, 1.28-39.83 ; P=.025$ )

ship between plaque enhancement and the presence of acute ischemic lesions in the territory of the MCA, this relationship did not survive multivariate logistic regression analysis.

The degree of stenosis in ICAD is known to be a reliable predictor of ischemic stroke. A prospective study conducted by the WASID trial group in 339 patients with ICAD revealed a strikingly higher prevalence of recurrent stroke in the territory of the stenotic segment in patients with severe stenosis than in patients with moderate stenosis. ${ }^{16}$ The fact that symptomatic plaques were more likely to be more severe than asymptomatic plaques in terms of the degree of stenosis introduces a simple bias that could have affected the results: A larger plaque mass is more likely to show greater enhancement or to make enhancement more obvious or detectable given the still-limited resolution of the technique. We were, therefore, not able to confirm that contrast enhancement of plaques is a predictive factor for stroke risk independent of the degree of stenosis. Our findings suggested that higher grades of stenosis had greater enhancement that was not causally related to infarction but was instead simply caused by stenosis. Therefore, in order for Gd plaque enhancement in ICAD to be used as a predictor of ischemic stroke, a prospective trial should be designed in which risk and control groups have similar degrees of stenotic severity, or enhancement should be assessed in patients with equivalently stenotic bilateral plaques in the same location in the setting of unilateral acute infarction.

There was a difference in plaque enhancement in relation to the degree of stenosis. This finding can be explained in 3 ways: First, the role of the vasa vasorum should be considered. Unlike extracranial arteries, which are surrounded by solid tissue, intracranial arteries have littleto-no vasa vasorum. ${ }^{24,25}$ This finding is consistent with the hypothesis that the vasa vasorum in the intracranial artery does not necessarily have an obligatory role in lesion development during the early stages of atherosclerosis. As atherosclerosis progresses to an advanced stage, the vasa vasorum develops in the proximal-to-distal segments. The vasa vasorum in the distal segments sometimes develops independently ${ }^{26}$ and may play an important role in plaque growth. This hypothetic process is supported by a postmortem study that reported that the vasa vasorum was more frequently developed in aged patients with severe atherosclerosis. ${ }^{25}$ Second, inflammatory changes that occur within plaques during plaque growth may also explain the strong enhancement of severe stenosis. A histopathologic-radiologic correlation study of the extracranial carotid artery showed that Gd enhancement was significantly associated with neovascularization, macrophages, and loose fibrosis within plaques. ${ }^{9}$ This finding explains the positive relationship between Gd enhancement and both the severity of stenosis and clinical symptoms. Third, CR may increase with increased wall thickness, regardless of inflammatory and neovascular changes, simply by virtue of the decreased volume averaging with the surrounding CSF with an increase in vessel wall thickness.

Weaknesses of this study were the low number of subjects and the retrospective design. The lack of both pathologic correlation and conventional angiography is another major limitation. Because the primary management for ICAD at our institution is medical therapy, pathologic confirmation or diagnostic angiography is strictly limited, even for symptomatic lesions. 


\section{CONCLUSIONS}

This study demonstrated that the enhancement of plaques in the intracranial artery was closely related to the severity of stenosis. This relationship may be due to different degrees of neovascularity or permeability according to plaque progression. Because stenotic degree may be simultaneously associated with plaque contrast enhancement on MCA and clinical symptoms, the previously reported relationship between plaque enhancement and stroke derived from univariate comparisons should be reconsidered. To settle the debate about the predictive value of plaque enhancement in the setting of intracranial atherosclerosis, further studies are required to compare symptomatic and asymptomatic subjects with similar degrees of stenosis severity.

\section{REFERENCES}

1. Chimowitz MI, Lynn MJ, Howlett-Smith H, et al. Comparison of warfarin and aspirin for symptomatic intracranial arterial stenosis. New Engl J Med 2005;352:1305-16

2. Wong KS, Huang YN, Gao S, et al. Intracranial stenosis in Chinese patients with acute stroke. Neurology 1998;50:812-13

3. O'Brien KD, Allen MD, McDonald TO, et al. Vascular cell adhesion molecule-1 is expressed in human coronary atherosclerotic plaques: implications for the mode of progression of advanced coronary atherosclerosis. J Clin Invest 1993;92:945-51

4. O'Brien KD, McDonald TO, Chait A, et al. Neovascular expression of E-selectin, intercellular adhesion molecule-1, and vascular cell adhesion molecule- 1 in human atherosclerosis and their relation to intimal leukocyte content. Circulation 1996;93:672-82

5. Moulton KS, Vakili K, Zurakowski D, et al. Inhibition of plaque neovascularization reduces macrophage accumulation and progression of advanced atherosclerosis. Proc Natl Acad Sci U A 2003;100:4736-41

6. Celletti FL, Waugh JM, Amabile PG, et al. Vascular endothelial growth factor enhances atherosclerotic plaque progression. Nat Med 2001;7:425-29

7. Yuan C, Kerwin WS, Ferguson MS, et al. Contrast-enhanced high resolution MRI for atherosclerotic carotid artery tissue characterization. J Magn Reson Imaging 2002;15:62-67

8. Kerwin WS, O'Brien KD, Ferguson MS, et al. Inflammation in carotid atherosclerotic plaque: a dynamic contrast-enhanced MR imaging study. Radiology 2006;241:459-68

9. Millon A, Boussel L, Brevet M, et al. Clinical and histological significance of gadolinium enhancement in carotid atherosclerotic plaque. Stroke 2012;43:3023-28

10. Lombardo A, Rizzello V, Natale L, et al. Magnetic resonance imaging of carotid plaque inflammation in acute coronary syndromes: a sign of multisite plaque activation. Int J Cardiol 2009;136:103-05
11. Millon A, Mathevet JL, Boussel L, et al. High-resolution magnetic resonance imaging of carotid atherosclerosis identifies vulnerable carotid plaques. J Vasc Surg 2013;57:1046-51

12. Skarpathiotakis M, Mandell DM, Swartz RH, et al. Intracranial atherosclerotic plaque enhancement in patients with ischemic stroke. AJNR Am J Neuroradiol 2013;34:299-304

13. Kim JM, Jung $\mathrm{KH}$, Sohn $\mathrm{CH}$, et al. Middle cerebral artery plaque and prediction of the infarction pattern. Arch Neurol 2012;69:1470-75

14. Vergouwen MD, Silver FL, Mandell DM, et al. Eccentric narrowing and enhancement of symptomatic middle cerebral artery stenoses in patients with recent ischemic stroke. Arch Neurol 2011;68:338-42

15. Klein IF, Lavallee PC, Mazighi M, et al. Basilar artery atherosclerotic plaques in paramedian and lacunar pontine infarctions: a high-resolution MRI study. Stroke 2010;41:1405-09

16. Kasner SE, Chimowitz MI, Lynn MJ, et al. Predictors of ischemic stroke in the territory of a symptomatic intracranial arterial stenosis. Circulation 2006;113:555-63

17. Jung JM, Kang DW, Yu KH, et al. Predictors of recurrent stroke in patients with symptomatic intracranial arterial stenosis. Stroke 2012;43:2785-87

18. Lee DK, Kim JS, Kwon SU, et al. Lesion patterns and stroke mechanism in atherosclerotic middle cerebral artery disease: early diffusion-weighted imaging study. Stroke 2005;36:2583-88

19. Bash S, Villablanca JP, Jahan R, et al. Intracranial vascular stenosis and occlusive disease: evaluation with CT angiography, MR angiography, and digital subtraction angiography. AJNR Am J Neuroradiol 2005;26:1012-21

20. Choi CG, Lee DH, Lee JH, et al. Detection of intracranial atherosclerotic steno-occlusive disease with $3 \mathrm{D}$ time-of-flight magnetic resonance angiography with sensitivity encoding at 3T. AJNR Am JNeuroradiol 2007;28:439-46

21. Kasner SE, Lynn MJ, Chimowitz MI, et al. Warfarin vs aspirin for symptomatic intracranial stenosis: subgroup analyses from WASID. Neurology 2006;67:1275-78

22. Samuels OB, Joseph GJ, Lynn MJ, et al. A standardized method for measuring intracranial arterial stenosis. AJNR Am J Neuroradiol 2000;21:643-46

23. Wattjes MP, Lutterbey GG, Harzheim M, et al. Imaging of inflammatory lesions at 3.0 Tesla in patients with clinically isolated syndromes suggestive of multiple sclerosis: a comparison of fluid-attenuated inversion recovery with T2 turbo spin-echo. Eur Radiol 2006;16:1494-500

24. Aydin F. Do human intracranial arteries lack vasa vasorum? A comparative immunohistochemical study of intracranial and systemic arteries. Acta Neuropathol 1998;96:22-28

25. Takaba M, Endo S, Kurimoto M, et al. Vasa vasorum of the intracranial arteries. Acta Neurochirurgica 1998;140:411-16

26. Atkinson JL, Okazaki H, Sundt TM Jr, et al. Intracranial cerebrovascular vasa vasorum associated with atherosclerosis and large thickwalled aneurysms. Surg Neurol 1991;36:365-69 